\title{
Retroperitoneal Schwannoma Associated with Neurofibromatosis Type I: Case Report
}

\author{
Jorge González-Tovar, Luis O. Flores-Salazar, Alondra Esparza-González and Gerardo E. Muñoz-Maldonado \\ General Surgery Service, "Dr. José Eleuterio González" University Hospital, Universidad Autónoma de Nuevo León, Monterrey, Mexico
}

\begin{abstract}
Schwannomas are tumors of neural origin. They represent $1 \%$ of all the tumors of the retroperitoneum. The majority are benign. However, up to $18 \%$ are malignant, and frequently with a low-grade differentiation in their histology. A 46-year-old female with neurofibromatosis type 1 who had right knee pain with irradiation to the ipsilateral leg was described. The diagnostic approach included lumbar magnetic resonance imaging showing a retroperitoneal tumor from $L 2$ to $L 3$. Despite the great development of therapeutic strategies, surgical excision seems to be the only one with curative potential. However, few patients are candidates for this treatment. In our case, we gave the patient a multidisciplinary treatment which leads to a good oncological outcome. Malignant degeneration of schwannomas is not uncommon. We propose the multidisciplinary approach for the diagnosis, the surgical procedure as well as follow-up for better outcomes.
\end{abstract}

Key words: Retroperitoneum. Schwannoma. Surgery.

\section{Introduction}

Schwannomas are tumors of neural origin that correspond to $1 \%$ of retroperitoneal tumors ${ }^{1}$. Most occur in young and middle-aged women and are commonly benign; only $5-18 \%$ are malignant and resemble a high-grade sarcoma ${ }^{2}$. In malignant presentations, the autosomal dominant association with neurofibromatosis type I or Von Recklinghausen ${ }^{3}$ disease is common. The definitive diagnosis is usually made after surgery. The most used imaging studies are ultrasound, computerized axial tomography, and magnetic resonance. This last one is the best to identify its nervous origin. The clinical picture depends on the affected spinal lev$\mathrm{el}$, and its management consists of complete resection as the only curative option ${ }^{1-3}$.

\section{Clinical Case}

A 46-year-old female patient with a diagnosis of neurofibromatosis type I began her condition 3 years before admission with pain in the right knee irradiated to the ipsilateral leg, associated with myalgia and paresthesia intermittently. So she went to an orthopedic consultation in which a magnetic resonance imaging of the thoracolumbar spine was requested, in which a tumor in the right retroperitoneal region measuring $10 \mathrm{~cm} \times 17 \mathrm{~cm}$ was found, with a spinal origin at the level of the second and third lumbar vertebrae (Fig. 1).

With these findings, the patient was referred to the general surgery service for surgical management. On admission, laboratory studies were performed within normal parameters, along with multidisciplinary

\section{Correspondence:}

Jorge González Tovar

E-mail: jrggzz@gmail.com CC BY-NC-ND license (http://creativecommons.org/licenses/by-nc-nd/4.0/).
Date of reception: 26-09-2017

Date of acceptance: 02-11-2017

DOI: 10.24875/RMU.M18000002
Available online: $30-05-2018$ Medicina Universitaria. 2018;20(1):35-37 www.medicinauniversitaria.org 


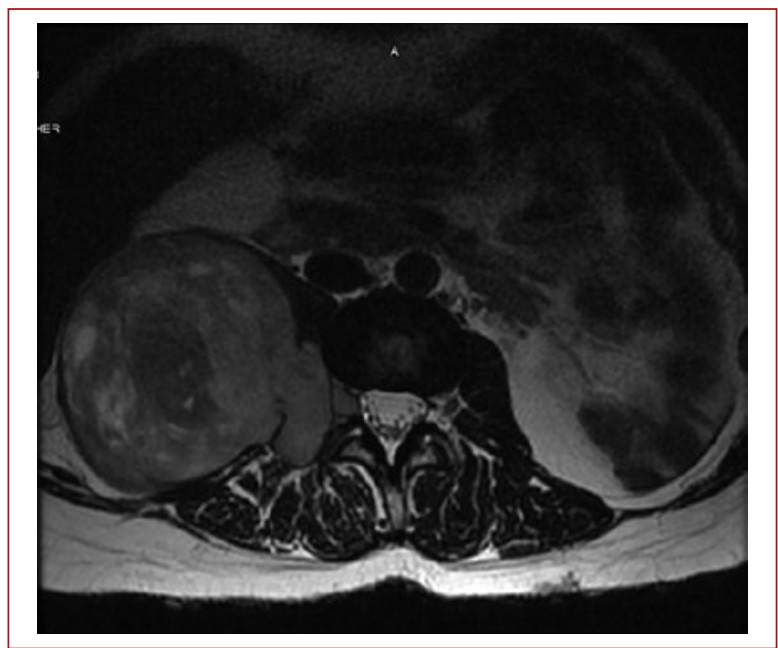

Figure 1. Magnetic resonance image is showing the retroperitoneal extension of the schwannoma and its origin in the spinal cord

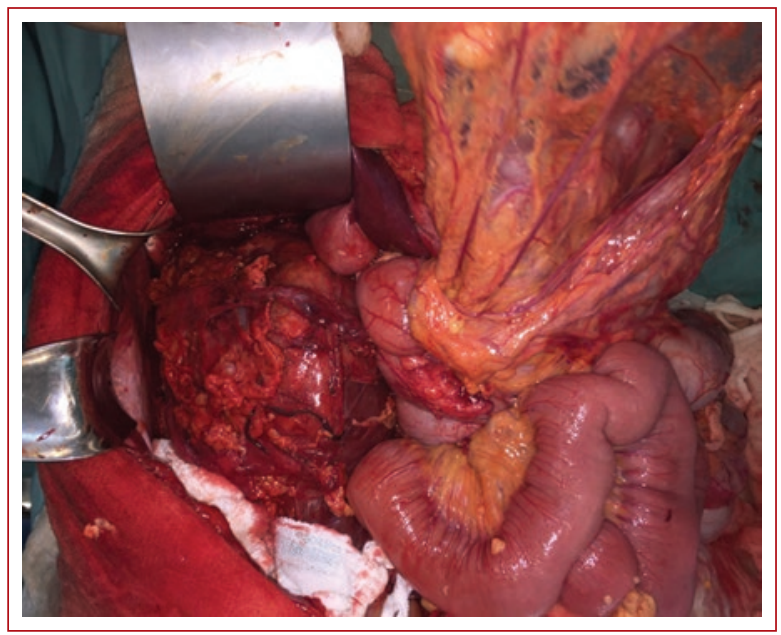

Figure 2. Image of the surgical bed showing the location and size of the schwannoma, as well as the neighboring structures involved

pre-operative evaluations with the oncology, urology, and neurosurgery services. During surgery, we found a right retroperitoneal tumor that measured $13 \mathrm{~cm} \times 17$ $\mathrm{cm}$, which was attached to the internal vena cava and the ureter. The tumor was dissected, finding its origin in the spinal cord at the level of the second lumbar space, the transverse processes were resected continuous to the tumor and completely resected (Figs. 2 and 3). The patient presented a favorable post-operative period and clinical improvement in the lower limb and was discharged on the $7^{\text {th }}$ post-operative day.

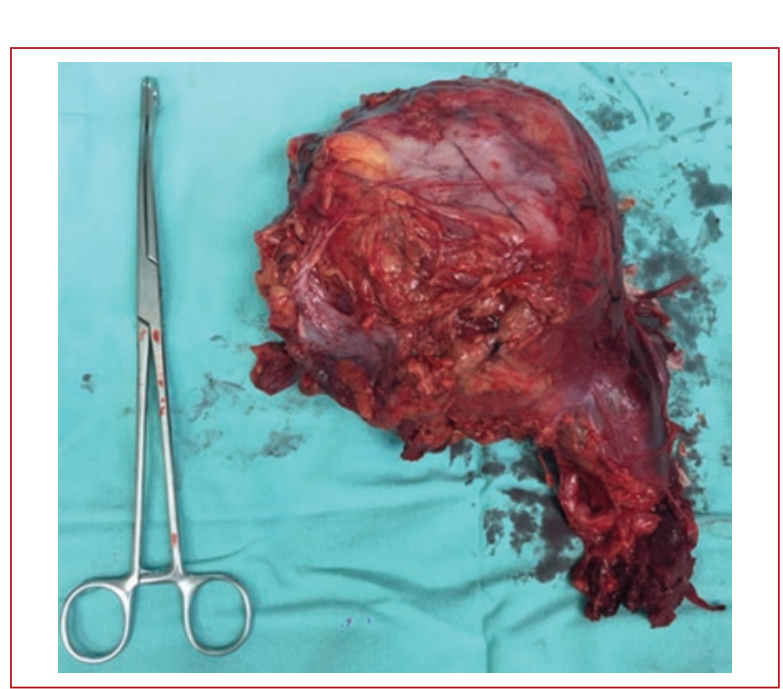

Figure 3. Image of a surgical piece with resection edges

The pathology report: a retroperitoneal/paravertebral tumor with a size of $17 \mathrm{~cm} \times 13.5 \mathrm{~cm}$, with a histological type of round cell sarcoma with characteristics of schwannoma, with a count of 52 mitoses in 10 high power fields, with a high associated histological grade to a $50 \%$ necrosis and without vascular invasion. Immunohistochemical stains were positive for myelin basic protein, CD99, vimentin, and S-100 and negative for smooth muscle actin, myogenin, CD34, pan-cytokeratin and HMB4. At present, the patient has evolved adequately, going to the Outpatient Surgery Consult where she was discharged definitively with follow-up by oncology, she is under oncological surveillance, denies neurological symptoms in the right limb and presents normal gait. Follow-up scans have been requested every 3 months and are negative for the recurrence of metastasis.

\section{Discussion}

The term schwannoma was first proposed by Masson in 1932. They are tumors originating in the nerve sheath. They correspond to $1 \%$ of retroperitoneal tumors and are usually benign since they only have a malignancy of $5-18 \%$. In the case of schwannomas of the lumbar region, these represent $25-30 \%$ of all spinal tumors $^{3,4}$. The statistics at our hospital is similar to this, with its frequent histology being liposarcomas.

Histologically, schwannomas are characterized by high cellularity areas called Antoni A regions, as well as myxoid matrix areas documented as Antoni $B^{5}$. In relation to immunohistochemistry; it is a tumor that is typically positive for S-100 stains, vimentin, and 
neuron-specific enolase, and negative for desmin, CD34, and CD117. Furthermore, a high mitotic index is also an indicator of malignancy ${ }^{6,7}$.

Our case fulfilled the immunohistochemistry criteria to be a schwannoma like tumor of a high histological grade. Regarding their clinical manifestation, they are slow growing masses and usually do not invade neighboring structures when it comes to benign lesions, so their clinical picture depends on the compression effect of the areas they directly affect. Usually, the symptoms are radiculopathy, paresis, paresthesia, diffuse abdominal pain, low back pain, and gastrointestinal disorders.

On the other hand, malignant schwannomas invade adjacent structures with a higher rate of recurrence ${ }^{7}$. The clinical presentation in our patient with neurapraxia of the right leg is typical of radiculopathy due to the involvement at the level of the second lumbar vertebra. During surgery, we found evidence of the tumor's adherence to the inferior vena cava corresponding to that described in malignant lesions. In general, the diagnosis is made through imaging studies. However, it can only be confirmed with histopathological study after resection. Magnetic resonance imaging is the best method to identify its nervous or spinal origin, where it was identified as an isointense mass in the $T 1$ phase and hyperintense in T2. However, radiological findings will hardly determine the behavior of the mass unless there is metastasis at a distance or invasion of neighboring structures 7,8 . There is the alternative of performing a biopsy or image-guided punctures with which a pre-operative histopathological diagnosis can be obtained, however, that still has little impact on the surgical management of the patient due to the poor response to chemo and radiotherapy that this type of tumor presents. The best form of management for these tumors, as well as the only curative opportunity, is complete surgical resection, in addition to the potential known complications of a biopsy such as hemorrhage, infection, and risk of tumor implantation. Once the diagnosis of a retroperitoneal tumor has been made, its surgical management should be not delayed, since success and its prognosis depends on this. Regarding the surgical approach, this will depend on the extent and compromise or lack thereof of the medullary canal. A prior approach can be made when the tumor appears in the presacral region with the purpose of manipulating the vascular structures. The posterior approach is reserved for when there is a compromise of the medullary canal ${ }^{8}$.
Recurrence rates are $75-90 \%$ at 3 months and increase if a complete resection was not performed ${ }^{9}$.

\section{Conclusion}

In spite of the fact that they are benign tumors most of the time, the malignant presentation with the involvement of neighboring structures is not infrequent. One option is the angioembolization of the tumor with the purpose of diminishing trans-operative bleeding, placing double $\mathrm{J}$ catheters to prevent lesions of the urinary tract in such a way that in these patients, it is important to perform a multidisciplinary evaluation and management because this has an impact on their prognosis. The definitive histopathological diagnosis should be made under strict immunohistochemical techniques with the purpose of directing the post-operative oncological treatment.

\section{Ethical disclosures}

Protection of human and animal subjects. The authors declare that no experiments were performed on humans or animals for this study.

Confidentiality of data. The authors declare that they have followed the protocols of their work center on the publication of patient data.

Right to privacy and informed consent. The authors have obtained the written informed consent of the patients or subjects mentioned in the article. The corresponding author is in possession of this document.

\section{References}

1. Machairiotis $N$, Zarogoulidis $P$, Stylianaki $A$, et al. Pelvic schwannoma in the right parametrium. Int J Gen Med. 2013;6:123-6.

2. Chen W, Dang C, Zhu K, Li K. Preoperative management of giant retroperitoneal schwannoma: a case report and review of the literature. Oncol Lett. 2016;11:4030-4.

3. Mastoraki A, Toska F, Tsiverdis I, et al. Retroperitoneal schwannomas: dilemmas in diagnostic approach and therapeutic management. J Gastrointest Cancer. 2013;44:371-4.

4. Moazam F, Roger B, Talbert J. Retroperitoneal malignant schwannoma: a case report. J Pediatr Surg. 1983;18:189-92.

5. Strauss D, Qureshi Y, Hayes A, Thomas JM. Management of bening retroperitoneal schwannomas: a single-center experience. Am J Surg. 2011;202:194-8.

6. Hughes M, Thomas J, Fisher C, Moskovic EC. Imaging features of retroperitoneal and pelvic schwannomas. Clin Radiol. 2005;60:886-93.

7. Goh B, Tan Y, Chung Y, Chow PK, Ooi LL, Wong WK. Retroperitoneal schwannoma. Am J Surg. 2006;192:14-8.

8. Theodosopoulos T, Stafyla V, Tsiantoula P, et al. Special problems encountering surgical management of large retroperitoneal schwannomas. World J Surg Oncol. 2008;6:107.

9. Singh M, Kumar L, Chejara R, et al. Diagnostic dilemma of a rare, giant retroperitoneal schwannoma. A case report and review of literature. Case Rep Oncol Med. 2014;1:1-5. 